\title{
ON DERIVATION OF THE DENSITY OF STATES FOR PERIODIC OPERATORS BY THE METHOD OF ASYMPTOTIC EXPANSION
}

\author{
K. D. CHEREDNICHENKO ${ }^{1}$ AND P. PADILLA ${ }^{2}$ \\ ${ }^{1}$ St John's College, Oxford OX1 3JP, UK \\ (cheredni@maths.ox.ac.uk) \\ ${ }^{2}$ IIMAS, Circuito Escolar, Ciudad Universitaria, 04510, \\ Mexico (pablo@mym.iimas.unam.mx)
}

(Received 27 July 2004)

\begin{abstract}
A new asymptotic approach is suggested for studying spectra of linear differential operators with periodic coefficients. The resulting formal recurrent procedure and its rigorous justification allow us to prove a classical theorem on the density of states in one dimension.
\end{abstract}

Keywords: Bloch waves; density of states; asymptotics

2000 Mathematics subject classification: Primary 34L20

Secondary 74J99; 78M35

\section{Introduction}

Asymptotic methods have been successfully used for more than a century in the quantitative study of spectra of ordinary and partial differential operators and there is a vast body of literature on the subject (see, for example, monographs $[\mathbf{5}, \mathbf{9}, \mathbf{1 1}, \mathbf{1 4}]$ and the references therein). Notwithstanding the amount of research done in the area, there are two distinct aspects of the problem that have, as a rule, been treated separately.

The underlying idea exploited in the first kind of setting is to try and find out how eigenvalues and the corresponding eigenspaces in a parametrized family of operators change upon a small perturbation of the parameters. This may lead to a more general approach whereby the solution to the corresponding eigenvalue problem is sought as an appropriate formal series (ansatz) whose terms are arranged by orders of smallness as the perturbation goes to zero. The leading term of the series thus represents the unperturbed eigenfunction, while the higher-order terms describe the perturbed behaviour of the eigenspace and depend on certain geometric characteristics of the considered family of operators. From a physical point of view, this corresponds to understanding how a change in the values of the input data or geometric parameters of the system will affect the energy flux of the output. In brief, this is the objective of what is called perturbation theory (the books $[\mathbf{9}, \mathbf{1 0}, \mathbf{1 4}]$ contain detailed descriptions of the method and a large number of examples). 
The second issue concerning spectra of differential operators is the distribution of eigenvalues. Thinking first of a problem with discrete spectrum $\left\{\lambda_{n}\right\}_{n=1}^{\infty}$, one would like to understand how the spectrum behaves as $n \rightarrow \infty$ (see $[\mathbf{1 1}]$ and numerous references therein). Another important case is given by operators whose spectra have so-called bandgap structure $\bigcup\left[a_{n}, b_{n}\right]$. If the intervals $\left(a_{n}, b_{n}\right)$ are disjoint, one might enquire about the asymptotics of the gap length $a_{n}-b_{n-1}$ as $n \rightarrow \infty$ (see $\left.[\mathbf{5}, \mathbf{1 4}]\right)$. Physical motivation for this sort of question comes from the necessity to understand high-frequency oscillation modes of a bounded region or high-frequency wave propagation in unbounded domains.

In this study we attempt at bringing together the two aspects of the subject outlined above, by suggesting a perturbation-type ansatz that also contains certain information about the distribution of eigenvalues. More precisely, consider a linear second-order ordinary differential operator with periodic coefficients. It is well known that its spectrum in $L^{2}(\mathbb{R})$ is purely continuous and has the band-gap structure mentioned above. At each internal point of the spectrum there are two quasi-periodic generalized eigenfunctions that form the fundamental system of solutions of the corresponding ordinary differential equation (ODE). They are often referred to as Bloch eigenfunctions, or Bloch waves, which originated in the work [1] (a good introduction to Bloch waves is also contained in [4]). The 'quasi-period' of each of the Bloch waves can be described in terms of the so-called quasi-momentum $\eta$. As the spectral parameter $\lambda$ is varied along the given band, the corresponding quasi-momentum $\eta(\lambda)$ sweeps the segment $[-\pi, \pi]$ in some way. The pre-image of the Lebesgue measure on $[-\pi, \pi]$ under the map $\eta(\lambda)$ is usually referred to as the integrated density of states. The derivative of this with respect to $\lambda$ is called the density of states. The reason for using such terminology is partly due to the fact that there is an alternative, equivalent, definition of the density of states, via passing to the limit in a sequence of spectral distribution functions for an 'approximating' family of operators on bounded intervals $[-N, N]$ as $N \rightarrow \infty$ (see, for example, [10]).

Using this as a starting point, we fix $\lambda=\lambda_{0}$ and suggest a version of a perturbation ansatz around a Bloch eigenfunction corresponding to $\lambda_{0}$. As is customary in the method of formal asymptotic expansion, this leads to a non-trivial sequence of recurrence relations on the higher-order terms of the ansatz. Following rigorous analysis ensures that the series in fact converges in a neighbourhood of the point $\lambda_{0}$. Choosing the value of the perturbation parameter in order to satisfy zero boundary conditions, we get an implicit equation for the Dirichlet eigenvalues that are sufficiently close to $\lambda_{0}$. This enables us to pass to a double limit as $N \rightarrow \infty$ and $\lambda \rightarrow \lambda_{0}$ and thus obtain an independent proof of equivalence of the two definitions of the density of states.

\section{Setting of the problem}

Consider the following sequence of eigenvalue problems,

$$
\begin{array}{r}
u^{\prime \prime}(x)+\lambda q(x) u(x)=0, \\
u(0)=u(N)=0,
\end{array}
$$

where $q(x)$ is a positive periodic continuous function (without loss of generality, the period is assumed to be equal to 1), $\lambda>0$ is the spectral parameter and $N$ is a positive 
integer. Problem (2.1), (2.2) describes eigenmodes of a string with mass density $q(x)$ whose ends are clamped at the points $x=0$ and $x=N$. We would like to study the distribution of the eigenvalues $\lambda$ around a given value $\lambda_{0}$ as $N \rightarrow \infty$. This leads to the classical notion of the density of states of the periodic operator in $(2.1)($ see $[\mathbf{7}, \mathbf{1 0}, \mathbf{1 3}])$.

\section{Formal asymptotic procedure}

With the above purpose in mind, we fix the length of the physical domain $N$ and look for the eigenfunctions $u(x)$ in the following formal asymptotic form as $\lambda \rightarrow \lambda_{0}$ :

$$
u(x) \sim \exp \left(\sum_{n=0}^{\infty}\left(\lambda-\lambda_{0}\right)^{n} S_{n}\left(x, \lambda_{0}\right)\right) .
$$

This ansatz can be viewed as an 'infinite-order Bloch wave', since its principal term coincides with the classical Bloch eigenfunction (cf. $[\mathbf{1}, \mathbf{4}]$ ). In the expansion $(3.1)$, the functions $S_{n}\left(x, \lambda_{0}\right)$ do not have to be periodic, but their derivatives with respect to $x$ are required to be 1-periodic in $x$. Henceforth, we will use the notation $S_{n}(x)$ along with $S_{n}\left(x, \lambda_{0}\right)$.

We first use formal substitution of the series (3.1) into Equation (2.1) to find a sequence of recurrence relations for the functions $S_{n}(x)$ as follows:

$$
\begin{aligned}
S_{0}^{\prime \prime}(x)+\left(S_{0}^{\prime}(x)\right)^{2}+\lambda_{0} q(x) & =0, \\
S_{1}^{\prime \prime}(x)+2 S_{1}^{\prime}(x) S_{0}^{\prime}(x)+q(x) & =0, \\
S_{n}^{\prime \prime}(x)+\sum_{i+j=n} S_{i}^{\prime}(x) S_{j}^{\prime}(x) & =0, \quad n \geqslant 2 .
\end{aligned}
$$

Clearly, the function $S_{0}(x)$ has to be complex, since Equation (3.2) does not have solutions among real periodic $S_{0}^{\prime}(x)$. More careful examination of (3.2) leads to the conclusion that this equation is solvable if and only if the value $\lambda_{0}$ belongs to one of the so-called Bloch bands* of the spectrum of the self-adjoint periodic operator in the $L^{2}(\mathbb{R})$ generated by Equation (2.1) (the related background is provided in [4]). In the case when (3.2) is solvable, the space of solutions of the ODE (2.1) is spanned by two quasi-periodic Bloch eigenfunctions, which correspond to two complex-conjugate solutions of Equation (3.2).

To solve the eigenvalue problem (2.1), (2.2), we must now ensure that zero boundary conditions (2.2) are satisfied. To this end, notice that real and imaginary parts of expression (3.1) can be chosen as the basis of the space of solutions of the ODE (2.1), i.e. the required eigenfunction $u(x)$ can be sought in the following form,

$$
\begin{aligned}
u(x) \sim A_{1} \exp ( & \left.\sum_{n=0}^{\infty}\left(\lambda-\lambda_{0}\right)^{n} \operatorname{Re}\left(S_{n}(x)\right)\right) \cos \left(\sum_{n=0}^{\infty}\left(\lambda-\lambda_{0}\right)^{n} \operatorname{Im}\left(S_{n}(x)\right)\right) \\
& +A_{2} \exp \left(\sum_{n=0}^{\infty}\left(\lambda-\lambda_{0}\right)^{n} \operatorname{Re}\left(S_{n}(x)\right)\right) \sin \left(\sum_{n=0}^{\infty}\left(\lambda-\lambda_{0}\right)^{n} \operatorname{Im}\left(S_{n}(x)\right)\right),
\end{aligned}
$$

* In Floquet theory, these bands are also referred to as stability intervals (see the monograph [5] for a discourse on Floquet theory). 
where $A_{1}$ and $A_{2}$ are constants that may depend on $\lambda$. Without loss of generality, we can set $S_{n}(0)=0, n \geqslant 0$, and infer consequently that $A_{1}=0$, due to the condition $u(0)=0$. Hence

$$
u(x) \sim A_{2} \exp \left(\sum_{n=0}^{\infty}\left(\lambda-\lambda_{0}\right)^{n} \operatorname{Re}\left(S_{n}(x)\right)\right) \sin \left(\sum_{n=0}^{\infty}\left(\lambda-\lambda_{0}\right)^{n} \operatorname{Im}\left(S_{n}(x)\right)\right),
$$

and the condition $u(N)=0$ leads to the equation

$$
\sum_{n=0}^{\infty}\left(\lambda-\lambda_{0}\right)^{n} \operatorname{Im}\left(S_{n}(N)\right)=m \pi
$$

where $m \in \mathbb{Z}$. The eigenfunction (3.5) is a (formal) linear combination of the two conjugate solutions of the form (3.1). We show next that each of the formal expansions (3.1) defines a valid function $u(x)$ that satisfies the ODE (2.1), before proceeding to the interpretation of condition (3.6).

\section{Rigorous convergence of the formal ansatz}

We would like to ensure that, under the formal procedure described above, the series in the right-hand side of (3.1) converges as $\lambda \rightarrow \lambda_{0}$ for any $x$, so that (3.5) defines a function $u(x, \lambda)$, which is twice differentiable with respect to $x$ and satisfies Equation (2.1). To this end, we prove the following statement.

Theorem 4.1. For any given value of $N$, the following three power series converge uniformly with respect to $x \in[0, N]$ and $\lambda$ in a certain neighbourhood of the point $\lambda_{0}$ :

$$
\sum_{n=0}^{\infty}\left|\lambda-\lambda_{0}\right|^{n}\left|S_{n}(x)\right|, \quad \sum_{n=0}^{\infty}\left|\lambda-\lambda_{0}\right|^{n}\left|S_{n}^{\prime}(x)\right|, \quad \sum_{n=0}^{\infty}\left|\lambda-\lambda_{0}\right|^{n}\left|S_{n}^{\prime \prime}(x)\right| .
$$

Proof. Note first that each of the Equations (3.4), being a first-order linear ODE on the function $S_{n}^{\prime}(x)$, can be rewritten as follows,

$$
\left(S_{n}^{\prime}(x) \exp \left(2 S_{0}(x)\right)\right)^{\prime}=-\exp \left(2 S_{0}(x)\right) \sum_{\substack{i+j=n \\ i, j \geqslant 1}} S_{i}^{\prime}(x) S_{j}^{\prime}(x),
$$

from which we obtain

$$
S_{n}^{\prime}(x)=-\exp \left(-2 S_{0}(x)\right)\left(\int_{0}^{x} \exp \left(2 S_{0}(\tau)\right) \sum_{\substack{i+j=n \\ i, j \geqslant 1}} S_{i}^{\prime}(\tau) S_{j}^{\prime}(\tau) \mathrm{d} \tau+C_{n}\right) .
$$


The constant $C_{n}$ in the above equation is found from the condition of periodicity of the function $S_{n}^{\prime}(x)$, thus giving

$$
\begin{aligned}
S_{n}^{\prime}(x)=- & \exp \left(-2 S_{0}(x)\right)\left(\int_{0}^{x} \exp \left(2 S_{0}(\tau)\right) \sum_{\substack{i+j=n \\
i, j \geqslant 1}} S_{i}^{\prime}(\tau) S_{j}^{\prime}(\tau) \mathrm{d} \tau\right. \\
& \left.+\left(\exp \left(2 S_{0}(1)-2 S_{0}(0)\right)-1\right)^{-1} \int_{0}^{1} \exp \left(2 S_{0}(\tau)\right) \sum_{\substack{i+j=n \\
i, j \geqslant 1}} S_{i}^{\prime}(\tau) S_{j}^{\prime}(\tau) \mathrm{d} \tau\right) .
\end{aligned}
$$

Before proceeding further with the proof of the theorem, we make the following observation adopted from the work [2].

Observation. For any $n \in \mathbb{N}$, the following inequality holds:

$$
\sum_{i+j=n} \frac{1}{(i+1)^{2}(j+1)^{2}} \leqslant \frac{16}{(n+2)^{2}} .
$$

Employing induction, we show next that there are $D, \alpha>0$ such that, for any $n \geqslant 1$, the following uniform estimate holds:

$$
\left|S_{n}^{\prime}(x)\right| \leqslant \frac{D^{n}}{\alpha(n+1)^{2}} .
$$

Trivially, since the function $S_{1}^{\prime}(x)$ is periodic, it is bounded and hence, for any $\alpha>0$, there is a value $D>0$ such that the bound (4.4) holds for any $x \in[0, N]$ when $n=1$. It is important that, since $S_{n}^{\prime}(x)$ is 1-periodic, the constant $D$ can be chosen independent of $N$. (Note that $D$ may still depend on $\lambda_{0}$.) Assume now that the inequality (4.4) is satisfied for any $n \leqslant l-1$, where $l \geqslant 2$ is a given index. We would like to get the estimate (4.4) for $n=l$. Clearly, due to periodicity of the function $S_{m}^{\prime}(x)$, it is sufficient to estimate it on the interval $[0,1]$. In view of formula $(4.2)$, the induction hypothesis and the above observation, there is a positive constant $M$, which is determined by the function $S_{0}(x)$, such that the following inequalities are valid:

$$
\left|S_{l}^{\prime}(x)\right| \leqslant M \sum_{\substack{i+j=l \\ i, j \geqslant 1}} \frac{D^{i}}{\alpha(i+1)^{2}} \frac{D^{j}}{\alpha(j+1)^{2}}<M \frac{16 D^{l}}{\alpha^{2}(l+2)^{2}} .
$$

Hence, if we choose $\alpha \geqslant 16 M$, then

$$
\left|S_{l}^{\prime}(x)\right|<\frac{D^{l}}{\alpha(l+1)^{2}},
$$

thus completing the induction step.

Inequalities (4.4), together with Equations (3.4), allow us to get suitable uniform estimates on the functions $S_{n}(x), S_{n}^{\prime \prime}(x)$. To this end, notice first that, due to the condition $S_{n}(0)=0$, we get $S_{n}(x)=\int_{0}^{x} S_{n}^{\prime}(\tau) \mathrm{d} \tau$. Hence, invoking (4.4),

$$
\left|S_{n}(x)\right| \leqslant N \max _{x \in[0, N]}\left|S_{n}^{\prime}(x)\right| \leqslant \frac{N D^{n}}{\alpha(n+1)^{2}} .
$$


Finally, Equations (3.4), estimate (4.4) and relation (4.3) imply that, for $n \geqslant 2$, the following inequalities hold:

$$
\left|S_{n}^{\prime \prime}(x)\right| \leqslant \sum_{\substack{i+j=n \\ i, j \geqslant 1}}\left|S_{i}^{\prime}(x)\right|\left|S_{j}^{\prime}(x)\right| \leqslant \sum_{\substack{i+j=n \\ i, j \geqslant 1}} \frac{D^{i}}{\alpha(i+1)^{2}} \frac{D^{j}}{\alpha(j+1)^{2}}<\frac{16 D^{n}}{\alpha^{2}(n+2)^{2}} .
$$

Inequalities (4.4)-(4.6) imply that, for any fixed value of $N$, the series (4.1) converge uniformly for $\left|\lambda-\lambda_{0}\right| \leqslant D^{-1}$ and $x \in[0, N]$.

Therefore, if $\left|\lambda-\lambda_{0}\right| \leqslant D^{-1}$, the exponent in (3.1) defines a function $u(x)$, which satisfies the differential equation in (2.1) due to the relations (3.2)-(3.4). As a byproduct, we get the following statement about analyticity of the function $S_{0}(x)$ with respect to $\lambda$.

Corollary 4.2. For any $x \in[0,+\infty)$, the series $\sum_{n=0}^{\infty}\left(\lambda-\lambda_{0}\right)^{n} S_{n}(x)$ defines a realanalytic function of $\lambda$, which coincides with $S_{0}(x, \lambda)$. Hence, for any $\lambda_{0}$ from the spectrum and every $x \in[0,+\infty)$, the following formula holds:

$$
S_{n}\left(x, \lambda_{0}\right)=\left.\frac{1}{n !} \frac{\partial^{n}}{\partial \lambda^{n}} S_{0}(x, \lambda)\right|_{\lambda=\lambda_{0}}
$$

\section{Density of states}

In this section we clarify how Equation (3.6) is related to the desired distribution of the eigenvalues.

Consider the Bloch eigenfunctions for the periodic operator in (2.1) corresponding to the eigenvalue $\lambda_{0}$ from the spectrum in the following form (cf. $\left.[4,5]\right)$ :

$$
u_{ \pm}\left(x, \lambda_{0}\right)=\exp \left(v\left(x, \lambda_{0}\right) \pm \mathrm{i}\left(w\left(x, \lambda_{0}\right)+\eta\left(\lambda_{0}\right) x\right)\right)
$$

In this expression and in what follows, for any $\lambda$ from the spectrum, $\eta(\lambda)$ denotes the value of the so-called dual variable, or quasi-momentum $\eta \in[-\pi, \pi]$ corresponding to the eigenvalue $\lambda$. In formula (5.1), the function $v\left(x, \lambda_{0}\right)$ is 1-periodic in $x$ and $w\left(x+1, \lambda_{0}\right)-$ $w\left(x, \lambda_{0}\right)=2 \pi l_{0}$ for some integer $l_{0}$ and any $x \in \mathbb{R}$. Thus the functions (5.1) are bounded solutions of the equation

$$
u^{\prime \prime}(x)+\lambda_{0} q(x) u(x)=0 .
$$

It is easy to see that, as we mentioned before, solvability of Equation (3.2) is equivalent to the existence of bounded solutions of (5.2) of the above type. If such solutions exist, i.e. the value $\lambda_{0}$ belongs to one of the bands of the spectrum, we can set either

$$
S_{0}^{\prime}\left(x, \lambda_{0}\right)=v^{\prime}\left(x, \lambda_{0}\right)+\mathrm{i}\left(w^{\prime}\left(x, \lambda_{0}\right)+\eta\left(\lambda_{0}\right)\right)
$$

or

$$
S_{0}^{\prime}\left(x, \lambda_{0}\right)=v^{\prime}\left(x, \lambda_{0}\right)-\mathrm{i}\left(w^{\prime}\left(x, \lambda_{0}\right)+\eta\left(\lambda_{0}\right)\right) .
$$


In what follows, it does not make a difference which of the two we use, and we consider $S_{0}^{\prime}\left(x, \lambda_{0}\right)=v^{\prime}\left(x, \lambda_{0}\right)+\mathrm{i}\left(w^{\prime}\left(x, \lambda_{0}\right)+\eta\left(\lambda_{0}\right)\right)$. The function $S_{0}\left(x, \lambda_{0}\right)$ can then be found along the following lines:

$$
\begin{aligned}
S_{0}\left(x, \lambda_{0}\right) & =\int_{0}^{x} S_{0}^{\prime}\left(\tau, \lambda_{0}\right) \mathrm{d} \tau \\
& =\int_{0}^{x}\left(v^{\prime}\left(\tau, \lambda_{0}\right)+\mathrm{i}\left(w^{\prime}\left(\tau, \lambda_{0}\right)+\eta\left(\lambda_{0}\right)\right)\right) \mathrm{d} \tau \\
& =v\left(x, \lambda_{0}\right)-v\left(0, \lambda_{0}\right)+\mathrm{i}\left(w\left(x, \lambda_{0}\right)-w\left(0, \lambda_{0}\right)+\eta\left(\lambda_{0}\right) x\right) .
\end{aligned}
$$

Hence, due to periodicity properties of the functions $v\left(x, \lambda_{0}\right)$ and $w\left(x, \lambda_{0}\right)$, equality (3.6) takes the form

$$
2 \pi l_{0} N+\eta\left(\lambda_{0}\right) N+\left(\lambda-\lambda_{0}\right) \operatorname{Im}\left(S_{1}\left(N, \lambda_{0}\right)\right)+R(N, \lambda)=m \pi, \quad m \in \mathbb{Z},
$$

where, due to estimates (4.5), we have $R(N, \lambda)=O\left(N\left(\lambda-\lambda_{0}\right)^{2}\right)$ as $\lambda \rightarrow \lambda_{0}$, uniformly with respect to $N$. By virtue of the corollary in the previous section, we get

$$
\operatorname{Im}\left(S_{1}\left(N, \lambda_{0}\right)\right)=\operatorname{Im}\left(\left.\frac{\partial S_{0}(N, \lambda)}{\partial \lambda}\right|_{\lambda=\lambda_{0}}\right)=N \eta^{\prime}\left(\lambda_{0}\right)
$$

and therefore Equation (5.3) can be written as follows:

$$
\lambda=\lambda_{0}-\frac{m \pi-2 \pi l_{0} N-\eta\left(\lambda_{0}\right) N}{N \eta^{\prime}\left(\lambda_{0}\right)}+\frac{R(N, \lambda)}{N \eta^{\prime}\left(\lambda_{0}\right)} .
$$

This allows us to infer that the number of eigenvalues of problem (2.1) found in the interval $\left(\lambda_{0}-\Delta \lambda, \lambda_{0}+\Delta \lambda\right)$ is equal to the number of integer values $m$ such that

$$
-\Delta \lambda<\frac{m \pi-2 \pi l_{0} N-\eta\left(\lambda_{0}\right) N}{N \eta^{\prime}\left(\lambda_{0}\right)}+\frac{R(N, \lambda)}{N \eta^{\prime}\left(\lambda_{0}\right)}<\Delta \lambda .
$$

It is easy to see now that (5.4) is equivalent to the bounds

$$
-\Delta \lambda+O\left((\Delta \lambda)^{2}\right)<\frac{m \pi-2 \pi l_{0} N-\eta\left(\lambda_{0}\right) N}{N \eta^{\prime}\left(\lambda_{0}\right)}<\Delta \lambda+O\left((\Delta \lambda)^{2}\right) .
$$

Before making the main statement, we introduce the following definition (see, for example, $[7])$.

Definition 5.1. The function $\rho\left(\lambda_{0}\right)$, defined for any $\lambda_{0} \in \mathbb{R}$ by the formula

$$
\rho\left(\lambda_{0}\right)=\lim _{\Delta \lambda \rightarrow 0}(2 \Delta \lambda)^{-1} \lim _{N \rightarrow \infty} N^{-1} \#\left\{\lambda: \lambda \text { eigenvalue of }(2.1),\left|\lambda-\lambda_{0}\right| \leqslant \Delta \lambda\right\},
$$

is called the density of states of the operator $\mathrm{d}^{2} / \mathrm{d} x^{2}+\lambda q(x)$ in $L^{2}(\mathbb{R})$.

It is convenient to use the bounds (5.5) to calculate the density of states in our case. As a result, we obtain the following classical theorem. 
Theorem 5.2. For any value $\lambda$ from the interior of the spectrum of the operator $\mathrm{d}^{2} / \mathrm{d} x^{2}+\lambda q(x)$ in $L^{2}(\mathbb{R})$, its density of states $\rho(\lambda)$ and the quasi-momentum $\eta(\lambda)$ are related by the formula

$$
\rho(\lambda)=\frac{\eta^{\prime}(\lambda)}{\pi} .
$$

Proof. Indeed, Equations (5.5) and (5.6) imply that

$$
\begin{aligned}
& \rho\left(\lambda_{0}\right)=\lim _{\Delta \lambda \rightarrow 0}(2 \Delta \lambda)^{-1} \lim _{N \rightarrow \infty} N^{-1} \#\left\{m \in \mathbb{Z}:-\Delta \lambda+O\left((\Delta \lambda)^{2}\right)\right. \\
& \left.<\frac{m \pi-2 \pi l_{0} N-\eta\left(\lambda_{0}\right) N}{N \eta^{\prime}\left(\lambda_{0}\right)}<\Delta \lambda+O\left((\Delta \lambda)^{2}\right)\right\} \\
& =\lim _{\Delta \lambda \rightarrow 0}(2 \Delta \lambda)^{-1} \lim _{N \rightarrow \infty} N^{-1} \#\left\{m \in \mathbb{Z}: 2 l_{0}+\frac{\eta\left(\lambda_{0}\right)}{\pi}-\frac{\eta^{\prime}\left(\lambda_{0}\right)}{\pi} \Delta \lambda+O\left((\Delta \lambda)^{2}\right)\right. \\
& \left.<\frac{m}{N}<2 l_{0}+\frac{\eta\left(\lambda_{0}\right)}{\pi}+\frac{\eta^{\prime}\left(\lambda_{0}\right)}{\pi} \Delta \lambda+O\left((\Delta \lambda)^{2}\right)\right\} \\
& =\lim _{\Delta \lambda \rightarrow 0}(2 \Delta \lambda)^{-1}\left(2 \frac{\eta^{\prime}\left(\lambda_{0}\right)}{\pi} \Delta \lambda+O\left((\Delta \lambda)^{2}\right)\right) \\
& =\frac{\eta^{\prime}\left(\lambda_{0}\right)}{\pi} \text {. }
\end{aligned}
$$

Note. The 'clamping points' in (2.2) were chosen to be at $x=0$ and $x=N$ merely for the sake of convenience. From a physical point of view, the choice $u\left(-\frac{1}{2} N\right)=u\left(\frac{1}{2} N\right)=0$ might seem more natural. Of course, our approach is invariant with respect to such translation of coordinates.

Theorem 5.2 is well known in the spectral theory of operators with periodic coefficients (see, for example, $[\mathbf{7}]$ ). The procedure described above suggests an alternative way for its derivation by means of a classical method of asymptotic expansion. The resulting system of recurrence relations contains essential information about the spectrum of the corresponding differential operator.

\section{Concluding remarks}

(1) The above asymptotic technique can be implemented in order to prove formula (5.7) in a number of other problems of mathematical physics. In particular, the above holds for the 'electric conduction' operator $(\mathrm{d} / \mathrm{d} x) a(x) \mathrm{d} / \mathrm{d} x+\lambda$, where $a(x)$ is a positive 1-periodic function representing conductivity of the material and for the Hill-Schrödinger operator $-\mathrm{d}^{2} / \mathrm{d} x^{2}+V(x)-\lambda$ with a periodic potential $V(x)$.

(2) One of the strengths of our method is that it is equally applicable in the case of other types of boundary conditions for the operator on the bounded interval. For example, one could consider periodic, skew-periodic or Sturm-Liouville boundary 
conditions at the endpoints of the segment $[0, N]$, and obtain the formula (5.7) in those settings as well (cf. $[\mathbf{6}, \mathbf{1 0}, \mathbf{1 3}])$.

(3) An asymptotic expansion of the type (3.1) was considered in the paper [2] in connection with a study of high-frequency eigenmodes of the equation for a string with rapidly oscillating periodic density,

$$
u^{\prime \prime}(x)+\nu q(x / \varepsilon) u(x)=0, \quad u(0)=u(1)=0,
$$

as $\varepsilon \rightarrow 0$. Making the change of variable $y=x / \varepsilon$ in (6.1), we arrive at the problem

$$
u^{\prime \prime}(y)+\nu \varepsilon^{2} q(y) u(y)=0, \quad u(0)=u\left(\varepsilon^{-1}\right)=0 .
$$

In the paper [2], the above ansatz is used to investigate the behaviour of the eigenvalues and the corresponding eigenfunctions when $\nu \varepsilon^{2} \rightarrow 0$; the same authors in [3] study the case $\nu \varepsilon^{2} \rightarrow \infty$. Thus our present work can also be viewed as an attempt to 'fill the gap' between these two extreme situations.

(4) The series (3.1) can be viewed as a 'local' analogue of the classical WKB-type expansion, which has been used to study the asymptotics of eigenfunctions for large $\lambda$ (see, for example, $[\mathbf{3}, \mathbf{8}, \mathbf{1 2}])$.

Acknowledgements. The authors are grateful to M. I. Belishev and N. E. Firsova for their valuable comments. K.D.C. acknowledges the generous financial support of St John's College, Oxford. Both authors are thankful to the Mathematical Institute of the University of Oxford for its hospitality.

\section{References}

1. F. BLOCH, Über die Quantenmechanik der Electronen in Kristalgittern, Z. Phys. 52 (1928), 555-600.

2. C. Castro And E. Zuazua, Low frequency asymptotic analysis of a string with rapidly oscillating density, SIAM J. Appl. Math. 60 (2000), 1205-1233.

3. C. Castro and E. Zuazua, High frequency asymptotic analysis of a string with rapidly oscillating density, Eur. J. Appl. Math. 11 (2000), 595-622.

4. C. Conca And M. Vanninathan, Homogenisation of periodic structures via Bloch decomposition, SIAM J. Appl. Math. 57 (1997), 1639-1659.

5. M. S. P. Eastham, The spectral theory of periodic differential equations (Scottish Academic Press, Edinburgh, 1973).

6. P. G. GRinevich and R. G. Novikov, Discrete spectrum for $n$-cell potentials, Rapport de Recherches no. 98/10-2, Université de Nantes, available at http://arxiv.org/pdf/math$\mathrm{ph} / 9811014$.

7. R. Johnson and J. Moser, The rotation number for almost periodic potentials, Commun. Math. Phys. 84 (1982), 403-438.

8. V. MARChENKO, Sturm-Liouville operators and applications (Birkhäuser, 1986).

9. V. Maz'Ya, S. Nazarov and B. PlamenevskiJ, Asymptotic theory of elliptic boundary value problems in singularly perturbed domains, vol. I (Birkhäuser, 2000).

10. M. Reed And B. Simon, Methods of modern mathematical physics, vol. IV, Analysis of operators (Academic, 1978). 
11. Yu. SAFAROV AND D. VASSILIEV, The asymptotic distribution of eigenvalues of partial differential operators, Translations of Mathematical Monographs, vol. 155 (American Mathematical Society, Providence, RI, 1997).

12. D. Shenk And M. A. Shubin, Asymptotic expansion of the state density and the spectral function of a Hill operator, Mat. USSR Sb. 56 (1987), 473-490.

13. M. A. Shubin, The spectral theory and the index of elliptic operators with almost periodic coefficients, Russ. Math. Surv. 34 (1979), 109-157.

14. E. C. TITCHMARsh, Eigenfunction expansions associated with second-order differential equations (Clarendon, Oxford, 1962). 